\title{
A WAVE EQUATION WITH DISCONTINUOUS TIME DELAY
}

\author{
JOSEPH WIENER \\ Department of Mathematics \\ The University of Texas - Pan American \\ Edinburg, Texas 78539 \\ and \\ LOKENATH DEBNATH \\ Department of Mathematics \\ University of Central Florida \\ Orlando, Florida 32816
}

(Received October 21, 1991)

\begin{abstract}
The influence of certain discontinuous delays on the behavior of the solutions of the wave equation is studied.
\end{abstract}

KEY WORDS AND PHRASES: Partial Differential Equation, Piecewise Constant Delay, Wave Equation, Boundary Value Problem, Fourier Method, Oscillation

1991 AMS SUBJECT CLASSIFICATION CODES. 35L05, 35L20, 35L50, 35L99.

\section{INTRODUCTION.}

Functional differential equations (FDE) with delay provide a mathematical model for a physical or biological system in which the rate of change of the system depends upon its past history. The theory of FDE with continuous argument is well developed and has numerous applications in natural and engineering sciences. This paper continues our earlier work [1-5] in an attempt to extend this theory to differential equations with discontinuous argument deviations. In these papers, ordinary differential equations with arguments having intervals of constancy have been studied. Such equations répresent a hybrid of continuous and discrete dynamical systems and combine properties of both differential and difference equations. They include as particular cases loaded and impulse equations, hence their importance in control theory and in certain biomedical models. Continuity of a solution at a point joining any two consecutive intervals implies recursion relations for the values of the solution at such points. Therefore, differential equations with piecewise constant argument (EPCA) are intrinsically closer to difference rather than differential equations. In [6] boundary value problems for some linear EPCA in partial derivatives were considered and the behavior of their solutions studied. The results were also extended to equations with positive definite operators in Hilbert spaces. In [7] initial value problems were studied for EPCA in partial derivatives. A class of loaded equations that arise in solving certain inverse problems was explored within the general framework of differential equations with piecewise constant delay. The purpose of the present note is to investigate the influence of terms with piecewise constant time on the behavior of the solutions, especially their oscillatory properties, of the wave equation. 


\section{SEPARATION OF VARIABLES IN SYSTEMS OF PDE.}

Consider the boundary value problem (BVP) consisting of the equation

$$
U_{t}(x, t)=A U_{x x}(x, t)+B U_{x x}(x,[t]),
$$

the boundary conditions

$$
U(0, t)=U(1, t)=O,
$$

and the initial condition

$$
U(x, 0)=U_{0}(x) .
$$

Here $U(x, t)$ and $U_{0}(x)$ are real $m \times m$ matrices, $A$ and $B$ are real constant $m \times m$ matrices, and [.] designates the greatest integer function. According to [6], a matrix $U(x, t)$ is called a solution of BVP (2.1)-(2.3) if it satisfies the conditions: (i) $U(x, t)$ is continuous in $G=[0,1] \times[0, \infty)$; (ii) $U$, and $U_{x x}$ exist and are continuous in $G$, with the possible exception of the points $(x, n)$, where one-sided derivatives exist $(n=0,1,2, \ldots)$; (iii) $U(x, t)$ satisfies Eq. (2.1) in $G$, with the possible exception of the points $(x, n)$, and conditions (2.2), (2.3).

Looking for the solution of (2.1) - (2.3) in the form

$$
U(x, t)=T(t) X(x)
$$

gives

$$
T^{\prime}(t) X(x)=A T(t) X^{\prime \prime}(x)+B T([t]) X^{\prime \prime}(x),
$$

whence

$$
(A T(t)+B T([t]))^{-1} T^{\prime}(t)=X^{\prime \prime}(x) X^{-1}(x)=-P^{2},
$$

which generates the BVP

$$
\begin{gathered}
X^{\prime \prime}(x)+P^{2} X(x)=O, \\
X(0)=X(1)=O
\end{gathered}
$$

and the equation with piecewise constant argument

$$
T^{\prime}(t)=-A T(t) P^{2}-B T([t]) P^{2} .
$$

The general solution of Eq. (2.5) is

$$
X(x)=\cos (x P) C_{1}+\sin (x P) C_{2}
$$

where

$$
\cos (x P)=\sum_{n=0}^{\infty} \frac{(-1)^{n} x^{2 n} P^{2 n}}{(2 n) !}, \sin (x P)=\sum_{n=0}^{\infty} \frac{(-1)^{n} x^{2 n+1} P^{2 n+1}}{(2 n+1) !}
$$

and $C_{1}, C_{2}$ are arbitrary constant matrices. From $X(0)=O$ we conclude that $C_{1}=O$, and the condition $X(1)=O$ enables us to choose $\sin P=O$ (although this is not the necessary consequence of the equation $\left.(\sin P) C_{2}=O\right)$. This can be written as $e^{i P}-e^{-i P}=O$ or $e^{2 i P}=I$. Assuming that all eigenvalues $p_{1}, p_{2}, \ldots, p_{m}$ of $P$ are distinct and $S^{-1} P S=\mathcal{D}=\operatorname{diag}\left(p_{1}, p_{2}, \ldots, p_{m}\right)$, we have $e^{2 i S D S^{-1}}=I$, whence $S e^{2 i D} S^{-1}=I$ and $e^{2 i D}=I$. Therefore, $\mathcal{D}=\operatorname{diag}\left(\pi j_{1}, \pi j_{2}, \ldots, \pi j_{m}\right)$, where the $j_{k}$ are integers, and $P=S \mathcal{D S} S^{-1}, P^{2}-S \mathcal{D}^{2} S^{-1}=$ $S \operatorname{diag}\left(\pi^{2} j_{1}^{2}, \pi^{2} j_{2}^{2}, \ldots, \pi^{2} j_{m}^{2}\right) S^{-1}, \sin (x P)=S \sin (x \mathcal{D}) S^{-1}=S \operatorname{diag}\left(\sin \pi j_{1} x, \ldots, \sin \pi j_{m} x\right) S^{-1}$. Furthermore, we can put

$$
P_{j}=\operatorname{diag}(\pi(m(j-1)+1), \ldots, \pi m j), \quad(j=1,2, \ldots)
$$

in (2.5) and obtain the following result.

THEOREM 2.1. There exists an infinite sequence of matrix eigenfunctions for BVP (2.5)

$$
X_{j}(x)=\sqrt{2} \operatorname{diag}(\sin \pi(m(j-1)+1) x, \ldots, \sin \pi m j x), \quad(j-1,2, \ldots)
$$

which is complete and orthonormal in the space $L^{2}[0,1]$ of $m \times m$ matrices, that is 


$$
\int_{0}^{1} X_{j}(x) X_{k}(x) d x=\left\{\begin{array}{l}
O, j \neq k \\
I, j=k
\end{array}\right.
$$

where $I$ is the identity matrix.

REMARK. The matrices $S X,(x) S^{-1}$ satisfy Theorem 2.1 for any nonsingular $S$.

THEOREM 2.2. Let $E(t)$ be the solution of the problem

$$
T^{\prime}(t)=-A T(t) P^{2}, T(0)=I
$$

and let

$$
M(t)=E(t)+(E(t)-I) A^{-1} B .
$$

If the matrix $A$ is nonsingular, then Eq. (2.6) with the initial condition $T(0)=C_{0}$ has on $[0, \infty)$ a unique solution

$$
T(t)=M(t-[t]) M^{[t]}(1) C_{0} .
$$

PROOF. On the interval $n \leq t<n+1$, where $n \geq 0$ is an integer, Eq. (2.6) turns into

$$
T^{\prime}(t)=-A T(t) P^{2}-B C_{n} P^{2}, \quad C_{n}=T(n)
$$

with the general solution

$$
T(t)=E(t-n) C-A^{-1} B C_{n} .
$$

At $t=n$ we have $C_{n}=C-A^{-1} B C_{n}$, whence $C=\left(I+A^{-1} B\right) C_{n}$ and $T(t)=\left(E(t-n)+(E(t-n)-I) A^{-1} B\right) C_{n}$, that is,

$$
T(t)=M(t-n) C_{n} .
$$

At $t=n+1$ we have $C_{n+1}=M(1) C_{n}$ and

$$
C_{n}=M^{n}(1) C_{0}
$$

Hence,

$$
T(t)=M(t-n) M^{n}(1) C_{0}
$$

which is equivalent to (2.11).

THEOREM 2.3. If $\|M(1)\|<1$, then $\|T(t)\|$ exponentially tends to zero as $t \rightarrow+\infty$.

EXAMPLE. For the scalar parabolic equation

$$
u_{t}(x, t)=a^{2} u_{x x}(x, t)+b u_{x x}(x,[t])
$$

we have $m=1$ and $P_{j}=\pi j$, according to (2.7). For Eq. (2.9), with $A=a^{2}$ and $P=P_{j}$, we have $E_{j}(t)=e^{-a^{2} x^{2} j^{2}}$ and $M_{j}(t)=e^{-a^{2} \pi^{2} j^{2} t}-\frac{b}{a^{2}}\left(1-e^{-a^{2} \pi^{2} j^{2}}\right)$. Hence, the inequality $\left|M_{j}(1)\right|<1$ is equivalent to

$$
-1<e^{-a^{2} x^{2} j^{2}}-\frac{b}{a^{2}}\left(1-e^{-a^{2} x^{2} j^{2}}\right)<1
$$

whence

$$
-a^{2}<b<a^{2}\left(1+e^{-a^{2} x^{2} j^{2}}\right)\left(1-e^{-a^{2} x^{2} j^{2}}\right) .
$$

Since the function $\left(1+e^{-t}\right) /\left(1-e^{-t}\right)$ is decreasing, all functions $T_{j}(t)$ exponentially tend to zero as $t \rightarrow \infty$ if and only if

$$
-a^{2}<b \leq a^{2} .
$$

If $b<-a^{2}$, then all $T_{j}(t)$ monotonically tend to infinity as $t \rightarrow \infty$; and if $b>a^{2}\left(1+e^{-a^{2} \pi^{2}}\right)\left(1-e^{-a^{2} x^{2}}\right)$, then all $T_{j}(t)$ are unbounded and oscillatory. For any $b>a^{2}$, there exists a positive integer $j_{0}$ such that the $T_{j}(t)$ 
are unbounded and oscillatory, for $j>j_{0}$. Indeed, letting $b=a^{2}+\varepsilon$ and solving the inequality

$$
a^{2}+\varepsilon>a^{2}\left(1+e^{-a^{2} \pi^{2} j^{2}}\right)\left(1-e^{-a^{2} x^{2} j^{2}}\right)
$$

gives

$$
e^{-a^{2} x^{2} j^{2}}<\varepsilon /\left(2 a^{2}+\varepsilon\right),
$$

which holds for any $\varepsilon>0$ and sufficiently large $j$ and implies $M_{j}(1)<-1$. If $b=-a^{2}$, then $M_{j}(t)=1$, $T_{j}(t)=T_{j}(0)$ and $u(x, t)=u_{0}(x)$, for all $t$. Therefore, the condition $|b| \leq a^{2}$ is necessary and sufficient for the series

$$
u(x, t)=\sum_{j=1}^{\infty} T_{j}(t) x_{j}(x)
$$

to be a solution of the scalar BVP (2.1) - (2.3), with $A=a^{2}$ and $B=b$, if $u_{0}(x)$ is three times continuously differentiable. The coefficients $T,(0)$ are given by

$$
T_{j}(0)=\int_{0}^{1} u_{0}(x) X_{j}(x) d x,
$$

where $X_{j}(x)=\sqrt{2} \sin (\pi j x)$ and $u_{0}(x) \in C^{3}[0,1]$ satisfies $u_{0}(0)=u_{0}(1)=0$.

THEOREM 2.4. The solution $T=O$ of Eq. (2.6) is globally asymptotically stable as $t \rightarrow+\infty$ if and only if the eigenvalues $\lambda_{r}$ of the matrix $M(1)$ satisfy the inequalities

$$
\left|\lambda_{r}\right|<1, r=1, \ldots, m \text {. }
$$

PROOF. There exists a nonsingular matrix $Q$ such that $M(1)=Q J Q^{-1}$, where $J$ is a diagonal or Jordan matrix with the diagonal elements $\lambda_{r}$. Hence, from (2.13) it follows that

$$
C_{n}=\left(Q J Q^{-1}\right)^{n} C_{0}=Q J^{n} Q^{-1} C_{0}
$$

or

$$
C_{n}=\sum_{r=1}^{k} Q_{r}(n) \lambda_{r}^{n}, k \leq m
$$

where the entries of the matrices $Q_{n}(n)$ are polynomials of degree not exceeding $k-1$. This implies $C_{n} \rightarrow O$ as $n \rightarrow \infty$ if and only if (2.15) holds, and the conclusion of the theorem follows from (2.12).

LEMMA. All entries of every solution of the equation

$$
T^{\prime}(t)=A_{1} T(t) A_{2}
$$

with constant $m \times m$ matrices $A_{1}$ and $A_{2}$, are linear combinations of terms $t^{k} \exp \left(\alpha_{i}^{(1)} \alpha_{j}^{(2)} t\right)$, where $\alpha_{i}^{(1)}$ and $\alpha_{j}^{(2)}$ are eigenvalues of $A_{1}$ and $A_{2}$ and $k$ is integer.

PROOF. Assume, for simplicity, that the eigenvalues of $A_{1}$ and $A_{2}$ are distinct, and let

$$
\begin{aligned}
& S_{1}^{-1} A_{1} S_{1}=\mathcal{D}_{1}=\operatorname{diag}\left(\alpha_{1}^{(1)}, \alpha_{2}^{(1)}, \ldots, \alpha_{m}^{(1)}\right), \\
& S_{2}^{-1} A_{2} S_{2}=\mathcal{D}_{2}=\operatorname{diag}\left(\alpha_{1}^{(2)}, \alpha_{2}^{(2)}, \ldots, \alpha_{m}^{(2)}\right),
\end{aligned}
$$

Then the substitution $T=S_{1} V$ changes (2.17) to

$$
V^{\prime}(t)=\mathcal{D}_{1} V(t) A_{2}
$$

and the substitution $V=W S_{2}^{-1}$ transforms (2.18) into

$$
W^{\prime}(t)=\mathcal{D}_{1} W(t) \mathcal{D}_{2} .
$$

The entries of $W(t)$ are $c_{i j} \exp \left(\alpha_{i}^{(1)} \alpha_{j}^{(2)} t\right)$, with arbitrary constants $c_{i j}$, and the completion of the proof follows 
from the formula $T=S_{1} W S_{2}^{-1}$. If some of the eigenvalues are multiple, polynomial factors will replace the constants $c_{i j}$.

THEOREM 2.5. If all eigenvalues of $A$ have positive real parts, $U_{0}(x) \in C^{3}[0,1]$, and $\left\|A^{-1} B\right\|<1$, then BVP (2.1) - (2.3) has a solution (2.14). This series and all its term-by-term derivatives converge uniformly.

PROOF. The functions $T_{j}(t)$ satisfy Eq. (2.6), with $P_{j}$ given by (2.7). Therefore, setting in the above lemma $A_{1}=-A, A_{2}=P_{j}^{2}$ and noting that $\operatorname{Re} \alpha_{i}^{(1)}<0, \alpha_{j}^{(2)}>0$, we conclude that $E_{j}(t) \rightarrow O$ as $j \rightarrow \infty$ and all $t>0$, where $E_{j}(t)$ is the solution of (2.9). Hence, (2.10) implies $M_{j}(t) \rightarrow-A^{-1} B$ as $j \rightarrow \infty$ and $\left\|M_{j}(t)\right\|<1$, for sufficiently large $j$, by virtue of the condition $\left\|A^{-1} B\right\|<1$. From the formula

$$
T_{j}(t)=M_{j}(t-[t]) M_{j}^{[t]}(1) C_{0 j}
$$

we note that $\left\|T_{j}(t)\right\| \rightarrow 0$ exponentially as $t \rightarrow \infty$, for sufficiently large $j$. Therefore, for any $\varepsilon>0$, there exist $t=t_{0}>0$ and $j=N$ such that $\left\|R_{N}(t)\right\|<\varepsilon$, for $t>t_{0}$, where $R_{N}(t)$ is the remainder of series (2.14) after the $N$ th term. For $0<t \leq t_{0}$ and large $j$, we have $\left\|T_{j}(t)\right\|<\left\|C_{0 j}\right\|$, and in this case the uniform convergence of series (2.14), together with its respective derivatives in $t$ and $x$, follows from the smoothness of the initial function $U_{0}(x)$ and the formula

$$
T_{j}(0)=C_{0 j}=\int_{0}^{1} U_{0}(x) X_{j}(x) d x .
$$

THEOREM 2.6. If all eigenvalues $\lambda_{r}$ of the matrix $M(1)$ are negative, where $M(t)$ is defined by (2.10), then each entry of every matrix solution of Eq. (2.6) is oscillatory and, more precisely, has a zero in each interval $n \leq t \leq n+1$, for sufficiently large $n$.

PROOF. Let $\tau^{(i s)}(t)$ denote an entry of a solution $T(t)$ of Eq. (2.6) and let $c_{n}^{(i s)}=\tau^{(i s)}(n)$. Then from (2.16) we get

$$
c_{n}^{(i s)}=\sum_{r=1}^{k} q_{r}^{(i s)}(n) \lambda_{r}^{n}, \quad c_{n+1}^{(i s)}=\sum_{r=1}^{k} q_{r}^{(i s)}(n+1) \lambda_{r}^{n+1},
$$

where $q_{r}^{(i s)}$ are the entries of the matrices $Q_{r}$. If $c_{n}^{(i s)}=0$, for infinitely many $n$, then $\tau^{(i s)}(t)$ is oscillatory. Assuming $c_{n}^{(i s)} \neq 0$, for all sufficiently large $n$, we conclude that

$$
\lim _{n \rightarrow \infty} c_{n+1}^{(i s)} / c_{n}^{(i s)}=\lambda_{\mu}<0
$$

(since the coefficients $q_{r}^{(i s)}(n)$ are polynomials of $n$ ), where $\lambda_{\mu}$ is one of the eigenvalues of $M(1)$. Hence, $c_{n+1}^{(i s)} / c_{n}^{(i s)}<0$, starting with some $n$, which implies that $\tau^{(i s)}(t)$ has a zero in each interval $n<t<n+1$, for sufficiently large $t$.

THEOREM 2.7. If the matrix $M(1)$ has a negative eigenvalue, then there exists an initial matrix $C_{0}$ such that the corresponding solution $T(t)$ of Eq. (2.6) has an oscillatory entry with a zero in each interval $n<t<n+1$, for sufficiently large $n$.

PROOF. Assume, for simplicity, that in the equation $M(1)=Q J Q^{-1}$ the matrix $J$ is diagonal, $J=\operatorname{diag}\left(\lambda_{1}, \lambda_{2}, \ldots, \lambda_{m}\right)$, and that $\lambda_{1}<0$. In the equation $C_{n}=Q J^{n} Q^{-1} C_{0}$ put $C_{0}=Q$; then $C_{n}=Q J^{n}$ and $c_{n}^{(1)}=\left(q_{11} \lambda_{1}^{n}, q_{21} \lambda_{1}^{n}, \ldots, q_{m 1} \lambda_{1}^{n}\right)$, where $c_{n}^{(1)}$ is the first column of $C_{n}$ and $\left(q_{11}, q_{21}, \ldots q_{m 1}\right)$ is the first column of $Q$. Since $Q$ is nonsingular, there exists an element $q_{i l} \neq 0$. Hence, $\lim c_{n+1}^{(i l)} / C_{n}^{(i)}=\lambda_{1}<0$ as $n \rightarrow \infty$, which proves that the entry $\tau^{(i)}(t)$ of the solution $T(t)$ has a zero in each interval $(n, n+1)$, for sufficiently large $n$.

Following [8], we can prove that if the matrix $M(1)$ has no positive eigenvalues and no eigenvalues with equal moduli, then each entry of every solution of Eq. (2.6) is oscillatory. On the other hand, the availability of positive eigenvalues for $M(1)$ implies the existence of nonoscillatory entries for $T(t)$. 
Separation of variables in the matrix equation with constant coefficients

$$
U_{1}(x, t)=A_{0} U(x, t)+A_{2} U_{x x}(x, t)+B_{0} U(x,[t])+B_{2} U_{x x}(x,[t])
$$

leads to (2.5) and to the EPCA

$$
T^{\prime}(t)=A_{0} T(t)-A_{2} T(t) P^{2}+B_{0} T([t])-B_{2} T([t]) P^{2},
$$

which can be also investigated by the above method.

\section{A SCALAR WAVE EPCA.}

Separation of variables in the equation with constant coefficients

$$
u_{t r}(x, t)=a^{2} u_{x x}(x, t)-b u_{x x}(x,[t])
$$

and boundary conditions (2.2) yields $X_{j}(x)=\sqrt{2} \sin (\pi j x)$ and leads to the EPCA

$$
T^{\prime \prime}{ }_{j}(t)+a^{2} \pi^{2} j^{2} T_{j}(t)=b \pi^{2} j^{2} T_{j}([t]) .
$$

For brevity, we omit the subindex $j$ and make the substitution $T^{\prime}(t)-V(t)$, which changes (3.2) to a vector EPCA

$$
w^{\prime}(t)=A w(t)+B w([t])
$$

where $w=\operatorname{col}(T, V)$ and

$$
A=\left(\begin{array}{cc}
0 & 1 \\
-a^{2} \pi^{2} j^{2} & 0
\end{array}\right), \quad B=\left(\begin{array}{cc}
0 & 0 \\
b \pi^{2} j^{2} & 0
\end{array}\right) .
$$

Eq. (3.3) on the interval $n \leq t<n+1$ becomes

$$
w^{\prime}(t)=A w(t)+B c_{n}, c_{n}=w(n)
$$

with the solution

$$
w(t)=M(t-n) c_{n},
$$

where

$$
M(t)=e^{A t}+\left(e^{A t}-I\right) A^{-1} B .
$$

Therefore, Eq. (3.3) with the initial condition $w(0)=c_{0}$ has on $[0, \infty)$ a unique solution given by the right-hand side of (2.11), where $M(t)$ is defined in (3.4).

THEOREM 3.1. For $b<0$, the solution $w=0$ of Eq. (3.3) is unstable.

PROOF. Computations show that

$$
e^{A t}=\cos (\omega t) I+\omega^{-1} \sin (\omega t) A
$$

and

$$
e^{A t}-I=\left(\begin{array}{ll}
\cos \omega t-1 & \omega^{-1} \sin \omega t \\
-\omega \sin \omega t & \cos \omega t-1
\end{array}\right)
$$

where $\omega=a \pi j$. Also,

$$
\left(e^{A t}-I\right) A^{-1} B=\left(\begin{array}{ll}
b(1-\cos \omega t) / a^{2} & 0 \\
b \omega \sin \omega t / a^{2} & 0
\end{array}\right) .
$$

Hence,

$$
M(t)=\left(\begin{array}{ll}
\cos \omega t+b a^{-2}(1-\cos \omega t) & \omega^{-1} \sin \omega t \\
\left(b a^{-2}-1\right) \omega \sin \omega t & \cos \omega t
\end{array}\right)
$$

and

$$
\operatorname{det} M(1)=1-\frac{b}{a^{2}}+\frac{b}{a^{2}} \cos \omega
$$


The condition $b<0$ implies $\operatorname{det} M(1)>1$ and shows that at least one of the eigenvalues $\lambda$ of $M(1)$ satisfies $|\lambda|>1$. Therefore $\|w(t)\| \rightarrow \infty$ as $t \rightarrow+\infty$, for some initial vector $c_{0} \neq 0$.

THEOREM 3.2. For $b>a^{2}$, the solution $w=0$ of Eq. (3.3) is unstable.

PROOF. Calculations yield

$$
\operatorname{det}(M(1)-\lambda)=\lambda^{2}-2\left(\cos \omega+\frac{b}{a^{2}} \sin ^{2} \frac{\omega}{2}\right) \lambda+1-\frac{b}{a^{2}}+\frac{b}{a^{2}} \cos \omega
$$

and the expressions $\lambda_{1}=s+d, \lambda_{2}=s-d$ for the eigenvalues $\lambda_{1}, \lambda_{2}$ of $M(1)$, where $s=\cos \omega+\frac{b}{a^{2}} \sin ^{2 \omega} \frac{\omega}{2}$, $d^{2}=\left(\frac{b}{a^{2}}-1\right) \sin ^{2} \omega+\frac{b^{2}}{a^{4}} \sin ^{4} \frac{\omega}{2}$. The condition $b>a^{2}$ shows that $d^{2}>0$ and $\lambda_{1}>1$. The latter inequality implies $\|w(t)\| \rightarrow \infty$ as $t \rightarrow+\infty$, for some initial vector $c_{0} \neq 0$.

THEOREM 3.3. The solution $w=0$ of Eq. (3.3) is asymptotically stable as $t \rightarrow+\infty$ if an only if

$$
0<b<a^{2} \text {, }
$$

and $\omega \neq 2 \pi n, n=0,1,2, \ldots$.

PROOF. The condition $d^{2}<0$, which means that the eigenvalues of $M(1)$ are complex, leads to

$$
\cos ^{2} \frac{\omega}{2}>b^{2} /\left(2 a^{2}-b\right)^{2},
$$

whence

$$
b<a^{2}\left(1-\tan ^{2} \frac{\omega}{4}\right) \text { or } b<a^{2}\left(1-\cot ^{2} \frac{\omega}{4}\right) .
$$

Since $\left|\lambda_{1}\right|=\left|\lambda_{2}\right|$ and $\operatorname{det} M(1)=\lambda_{1} \lambda_{2}$, the inequality $\left|\lambda_{1}\right|<1$ is equivalent to $\operatorname{det} M(1)<1$, that is, to $b>0$. Therefore, in the case of complex eigenvalues, a criterion for asymptotic stability is $0<b<\max \left(a^{2}\left(1-\tan ^{2} \frac{\omega}{4}\right), a^{2}\left(1-\cot ^{2} \frac{\omega}{4}\right)\right)$. The inequality $d^{2}>0$ in the case of distinct real eigenvalues leads to $b>\max \left(a^{2}\left(1-\tan ^{2} \frac{\omega}{4}\right), a^{2}\left(1-\cot ^{2} \frac{\omega}{4}\right)\right)$, and the inequalities $\lambda_{1}<1, \lambda_{2}>-1$ yield $b<a^{2}$. Hence, in this case a criterion of asymptotic stability is

$$
\max \left(a^{2}\left(1-\tan ^{2} \frac{\omega}{4}\right), a^{2}\left(1-\cot ^{2} \frac{\omega}{4}\right)\right)<b<a^{2} .
$$

Finally, if $b=\max \left(a^{2}\left(1-\tan ^{2} \frac{\omega}{4}\right), a^{2}\left(1-\cot ^{2} \frac{\omega}{4}\right)\right)$, then $d=0$ and $\lambda_{1}=\lambda_{2}=\cos \omega+b a^{-2} \sin ^{2} \omega / 2$ whence $\cos \omega<\lambda_{1}<\cos ^{2} \omega / 2$ and $\left|\lambda_{1}\right|<1$. According to (2.15), this implies asymptotic stability and completes the proof of criterion (3.5).

REMARK 1. If $b=a^{2}$, then $\lambda_{1}=1, \lambda_{2}=\cos \omega$, and the solutions of (3.3) are bounded but not asymptotically stable.

REMARK 2. In Theorems 3.1 and 3.2 it was implicitly assumed that $\omega \neq 2 \pi n$. If $\omega=2 \pi n$, then $\lambda_{1}=\lambda_{2}=1$, which leads to the existence of unbounded solutions for (3.3).

COROLLARY 1. If the coefficient $a$ is irrational, then (3.5) is a criterion of asymptotic stability of the solutions to (3.2) for all $j$.

PROOF. Recalling that $\omega=\omega_{j}=a \pi j$, we note that the equality $a \pi j=2 \pi n$ is impossible for any irrational $a$.

COROLLARY 2. For any rational $a$, there exist infinitely many integers $j$ such that the corresponding solutions $w_{j}(t)$ of (3.3) are unbounded.

PROOF. The equation $\omega_{j}=2 \pi n$ implies $j=2 n / a$, and $j$ will be an integer for infinitely many integers

$n$. 
THEOREM 3.4. Each component of every solution of Eq. (3.3) oscillates if and only if either of the following conditions holds true:

(ii) $\max \left(a^{2}\left(1-\tan ^{2} \frac{\omega}{4}\right), a^{2}\left(1-\cot ^{2} \frac{\omega}{4}\right)\right)<b<\frac{a^{2}}{2 \sin ^{2} \frac{\omega}{2}}$ and $\cos \omega<-1 / 2$.

PROOF. Hypothesis (i) means that the eigenvalues of $M(1)$ are complex, and in this case all nontrivial solutions of (3.3) oscillate componentwise. In the case of real eigenvalues, we require $d^{2}>0$ and $\lambda_{1}=s+d<0$, which also implies $\lambda_{2}=s-d<0$, where $s$ and $d$ are defined in Theorem 3.2. The condition $d^{2}>0$ leads to the left-side inequality in (ii), and the condition $\lambda_{1}<0$ yields the right-side inequality in (ii). Finally, the restriction $\cos \omega<-1 / 2$ arises from the comparison of the inequalities $s<0$ and $\lambda_{1}<0$.

REMARK 3. The restriction $\cos \omega<-1 / 2$ implies $a^{2} / 2 \sin ^{2}(\omega / 2)<2 a^{2} / 3$.

In conclusion, it is worth noting that the asymptotic properties of Eq. (3.2) depend on the algebraic nature of the coefficient $a$. For $b<0$, all solutions of Eq. (3.2) are unstable and oscillatory; for $b>a^{2}$ all solutions of Eq. (3.2) are unstable and nonoscillatory. These two cases hold true for both rational and irrational values of $a$. For

$$
0<b<\max \left(a^{2}\left(1-\tan ^{2} \frac{\omega}{4}\right), a^{2}\left(1-\cot ^{2} \frac{\omega}{4}\right)\right),
$$

all solutions of (3.2) are asymptotically stable and oscillatory, provided that $\omega \approx 2 \pi n$. However, as indicated in Corollary 2 , for any rational $a$, there exist infinitely many integers $j$ such that $\omega_{j}=2 \pi n$, which leads to the existence of unbounded solutions for (3.2). Furthermore, since $\omega=\omega_{j}=a \pi j$, the inequality $\cos \omega<-1 / 2$ breaks down for infinitely many integers $j$. Therefore, under hypothesis (ii) of Theorem 3.4, there are infinitely many solutions of Eq. (3.2) which are asymptotically stable and oscillatory, as well as infinitely many solutions which are asymptotically stable and nonoscillatory $(\omega \neq 2 \pi n)$. Also, for $\omega \approx 2 \pi n$ and $a^{2} / 2 \sin ^{2}(\omega / 2)<b<a^{2}$, the solutions of (3.2) are asymptotically stable and nonoscillatory. Problems of this nature deserve further investigation.

ACKNOWLEDGEMENT. This research was partially supported by U.S. Army Grant DAAL03-89G-0107, NASA Grant NAG 9-553, and by the University of Central Florida.

\section{REFERENCES}

1. WIENER, J. Differential equations with piecewise constant delays, in Trends in the Theory and Practice of Nonlinear Differential Equations, Lakshmikantham, V. (editor), Marcel Dekker, New York, 1983, 546-552.

2. COOKE, K. L. and WIENER, J. Retarded differential equations with piecewise constant delays, J. Math. Anal. Appl. 99(1), (1984), 264-297.

3. SHAH, S. M. and WIENER, J. Advanced differential equations with piecewise constant argument deviations, Internat. J. Math. \& Math. Sci. 6(4), (1983), 671-703.

4. COOKE, K. L. and WIENER, J. Neutral differential equations with piecewise constant argument, Bolletino Unione Matematica Italiana 7 (1987), 321-346.

5. COOKE, K. L. and WIENER, J. An equation alternately of retarded and advanced type, Proc. Amer. Math. Soc. 99 (1987), 726-732.

6. WIENER, J. Boundary-value problems for partial differential equations with piecewise constant delay, Internat. J. Math. \& Math. Sci. 14 (1991), 301-321.

7. WIENER, J. and DEBNATH, L. Partial differential equations with piecewise constant delay, Internat. J. Math. \& Math. Sci. 14 (1991), 485-496.

8. WIENER, J. and COOKE, K. L. Oscillations in systems of differential equations with piecewise constant argument, J. Math. Anal. Appl. 137(1), (1989), 221-239. 


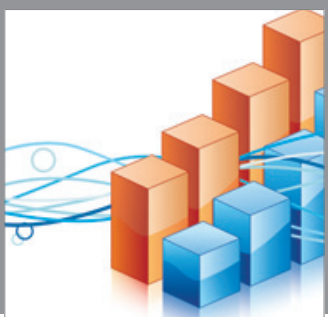

Advances in

Operations Research

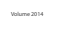

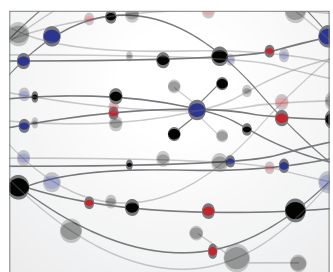

\section{The Scientific} World Journal
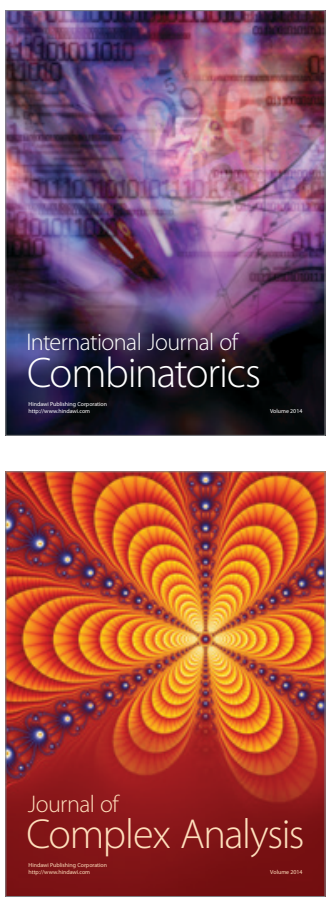

International Journal of

Mathematics and

Mathematical

Sciences
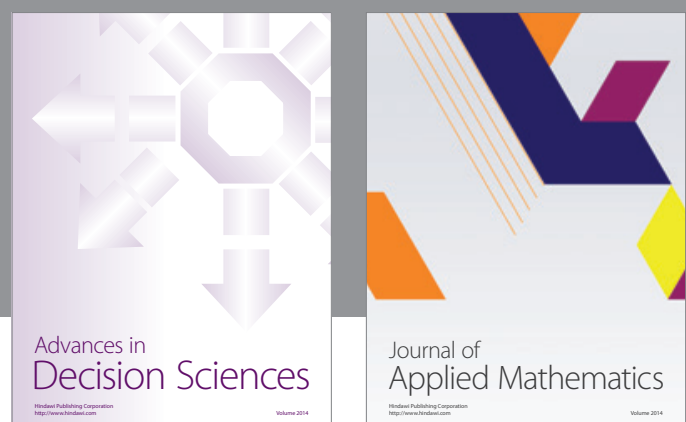

Journal of

Applied Mathematics
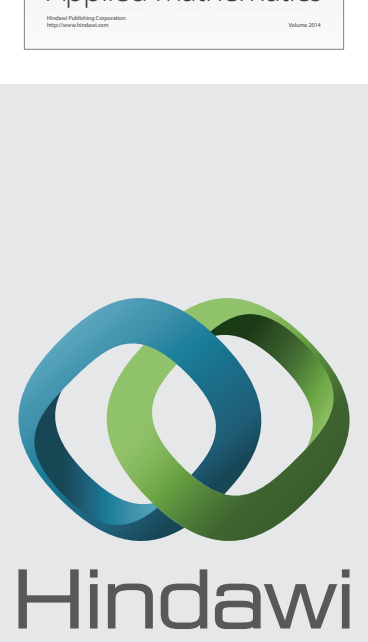

Submit your manuscripts at http://www.hindawi.com
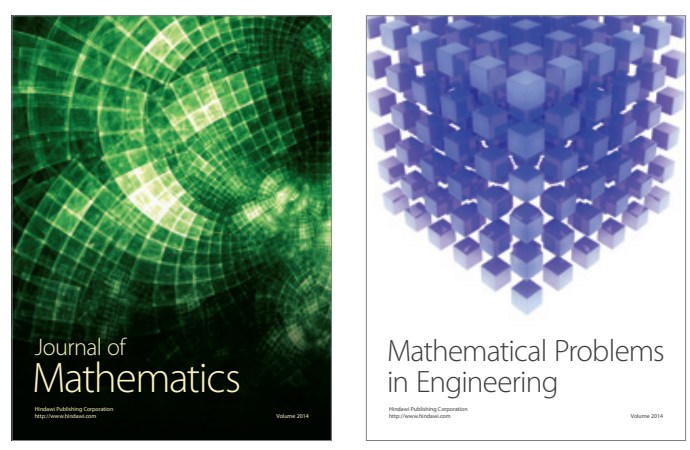

Mathematical Problems in Engineering
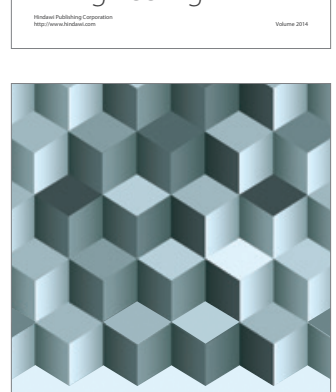

Journal of

Function Spaces
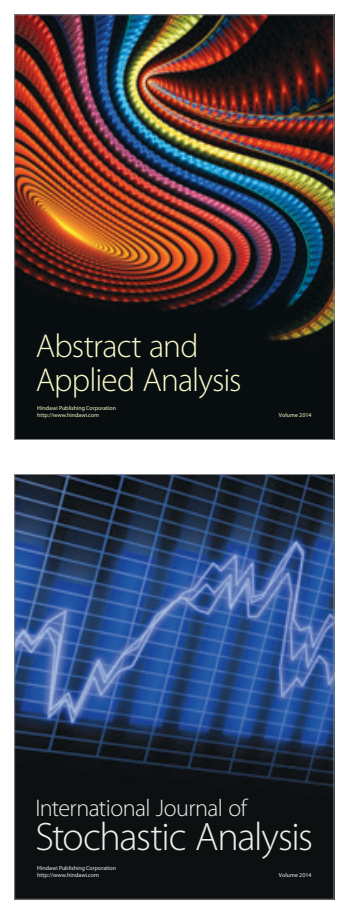

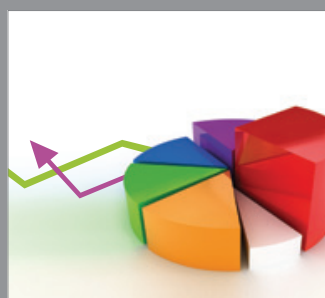

ournal of

Probability and Statistics

Promensencen
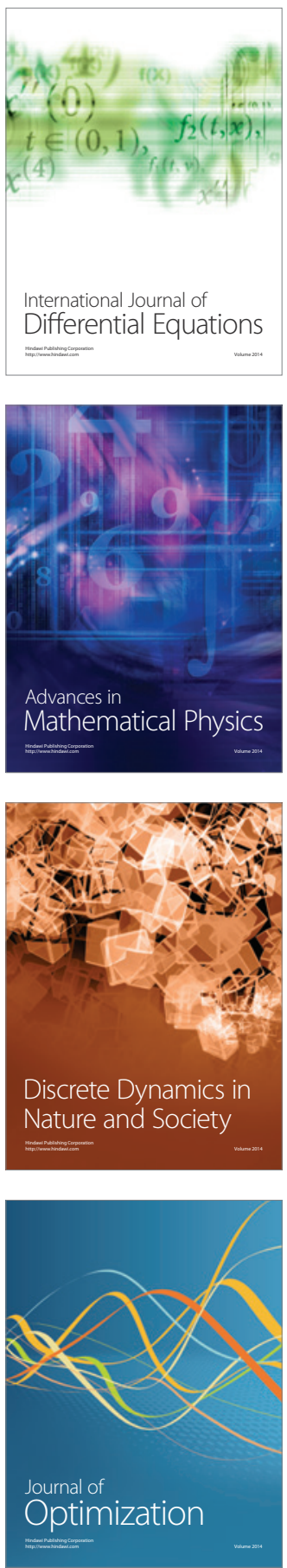